\title{
Gesundheitskosten: Mythen und Fakten
}

\section{Daniel Mahler}

Dr. med., Gruppe «Strategie und Kommunikation» der Schweizerischen Gesellschaft für Dermatologie und Venerologie (SGDV)

Um die Themen «Dämpfung der Kosten im Gesundheitswesen» sowie «Limitierung des Krankenkassenprämienwachstums» kommt rund ein Jahr vor den eidgenössischen Wahlen im Herbst 2019 keine Partei und kein Politiker herum. Die folgende Analyse versucht einige Mythen zu entkräften und auf bisher weniger beachtete Fakten hinzuweisen.

Die Gesundheitskosten stiegen im Jahre 2016 auf über 80 Milliarden Franken, der Anstieg von 3,8\% lag im Durchschnitt der Vorjahre. Bei den ambulant tätigen Ärztinnen und Ärzten lag das Wachstum mit 1,5\% unter dem 5-Jahres-Trend, andere ambulante Leistungserbringer wiesen mit über 10\% einen überdurchschnittlichen Kostenanstieg auf [1].

Der Mehrjahresvergleich der Gesundheitskosten in Prozent des Bruttoinlandproduktes (BIP) ist relevant. Kaum erwähnt wird allerdings, dass die Berechnung der Gesundheitskosten seit dem Jahre 2014 auf einer neuen Grundlage (OECD-Methodik) basiert, so dass zumindest ein Teil des Anstiegs (rund 3 Mrd. Franken bzw. ca. 0,5\% des BIP) berechnungstechnischer Natur ist [2].

\section{Qualität erhalten statt Leistungen rationieren}

Deutlicher als bislang sollte darauf hingewiesen werden, dass wir in der Schweiz erwiesenermassen eines der weltweit besten Gesundheitssysteme sowie eine der höchsten Lebenserwartungen aufweisen [3-5]. Dies wird mit einer nur unwesentlich über dem europäischen Durchschnitt liegenden Ärztedichte erreicht [3]. Aufgrund der zu geringen Anzahl von in der Schweiz ausgebildeten Ärztinnen und Ärzten weist unser Land zudem einen sehr hohen Anteil (etwa 1/3) an ausländischen Fachkräften auf [6].

Die allgemeine Lebenszufriedenheit, welche in der Schweiz sehr hoch ist, hängt auch vom Gesundheitszustand ab $[7,8]$. Bekannt ist, dass eine Bevölkerung mit hohem Wohlstandsniveau besonderen Wert auf eine gute Gesundheitsversorgung legt. Trotzdem vergleicht eine durch den Bundesrat beauftragte Expertengruppe unser hoch entwickeltes Gesundheitswesen mit Ländern wie Deutschland und Frankreich, in denen das System schlechter ist, die Leistungen rationiert werden (Globalbudgets) und eine Zweiklassenmedizin (Deutschland: Kassen- und Privatpatienten) herrscht $[4,9]$.

\section{Haushaltsausgaben für Dienstleistungen steigen generell}

Es findet bis dato zu wenig Beachtung, dass sich unsere Gesellschaft als Ganzes die Gesundheitskosten gut leisten kann [10]. Während die durchschnittlichen Ausgaben für materielle Güter stetig abnehmen, liegt es in der Natur der Sache, dass die Kosten in den Dienstleistungssektoren steigen. Im verhältnismässig wenig automatisierten Gesundheitswesen sind Produktivitätssteigerungen limitiert und gehen häufig mit einem Qualitätsverlust einher. Ein kürzlich erschienener Artikel in der Schweizerischen Ärztezeitung [11] beschreibt dieses als «baumolsche Kostenkrankheit» bezeichnete Phänomen eindrücklich. Aktuell betragen die Krankenkassenprämien etwa 5,9\% des durchschnittlichen Haushaltsbruttoeinkommens, dies entspricht einem Anstieg von 0,4 Prozentpunkten in den letzten 10 Jahren [12]. In Deutschland bezahlt der Bürger 14,6\% seines Einkommens an die Krankenversicherung [13] für ein Gesundheitssystem mit längeren Wartefristen und schlechterem Outcome [4].

Dass insbesondere Haushalte mit tiefem Einkommen unter der hohen Prämienlast leiden, ist unbestritten. Die Ursache dieses Missstandes ist in der Lastenverteilung zu suchen und nicht in den Kosten per se. Deswegen müssen für die Lösung andere, strukturelle Massnahmen als regulatorische hinzugezogen werden, dies 
auch angesichts der trotz Prämienverbilligung eher tiefen staatlichen Beteiligung an den Gesundheitskosten im internationalen Vergleich [3].

Ein oft angeführtes Argument ist, dass angeblich 20\% der Gesundheitsleistungen unnütz bzw. sogar schädlich seien [14]. Demgegenüber stehen die seit 2014 laufende Selbstregulierungsinitiative "Smarter Medicine» sowie die Bemühungen der Fachgesellschaften zur verstärkten Entwicklung von Guidelines, welche auf eine Verbesserung der Behandlungsqualität hinzielen $[15,16]$.

\section{Ein Prämientreiber, der gerne vergessen geht}

Der Weg, mit der Strategie "ambulant vor stationär» die Gesundheitskosten als Ganzes zu senken, ist richtig. Allerdings hat dieses Vorgehen einen Pferdefuss: Aufgrund unterschiedlicher Kostenverteilschlüssel setzt die Verlagerung von "stationär» zu «ambulant» zwar richtige Anreize zu tieferen Gesundheitsausgaben der öffentlichen Hand. Zugleich nimmt sie aber eine Fehlentwicklung in Kauf, die letztlich zu höheren Krankenkassenprämien führt [17]. Die laufenden politischen Bemühungen rund um die Einführung einer einheitlichen Finanzierung von ambulanten und stationären Behandlungen (EFAS) haben das grösste Potential, dieses Problem künftig zu vermindern oder gar zu verhindern [18].

\section{Fakten zu Löhnen der Ärztinnen und Ärzte}

Ein beliebter Mythos sind die angeblich überrissenen Löhne der Ärzteschaft. Statt Fakten zieht man Einzelfälle heran, verwechselt Umsatz mit Gewinn und bringt so die gesamte, seriös arbeitende Ärzteschaft in Misskredit. Die kürzlich publizierte MAS-Erhebung des Bundesamtes für Statistik (BfS) zeigt, dass das durchschnittliche Nettoeinkommen rund 155000 Franken beträgt [19]. Gemäss der Studie werden von 100 Franken der Gesamteinkünfte von als Einzelunternehmen organisierten Arztpraxen und ambulanten Zentren durchschnittlich 70 Franken für die Praxisbetriebskosten benötigt. Bei Arztpraxen und ambulanten Zentren mit einer anderen Rechtsform fliessen sogar 90 von 100 Franken in die Deckung des Aufwandes.

Die Ärztelöhne im ambulanten Bereich sind seit den 90er Jahren nicht mehr den aktuellen wirtschaftlichen Gegebenheiten (Anstieg der Praxisbetriebskosten, Teuerungsausgleich) angepasst worden. Während andere Zweige im Gesundheitswesen nicht davon betroffen sind, gilt das Gebot der Kostenneutralität damit für die Ärztinnen und Ärzte faktisch schon seit über 20 Jahren.

Selbstverständlich ist es zu vermeiden, dass über die obligatorische Krankenpflegeversicherung ungerechtfertigt hohe Umsätze erreicht werden, und gewisse Lohnunterschiede sind anzupassen. Dennoch muss das wirtschaftliche Führen einer Arztpraxis mit einem angemessenen Einkommen gewährleistet sein und Effizienz sollte sich weiterhin lohnen. Einen grossen Schritt in diese Richtung macht das Projekt TARCO, das aktuell mit den Tarifpartnern verhandelt wird [22].

\section{Demografische Entwicklung als Kostentreiber}

Das zukünftige Patentrezept scheint das sogenannte Globalbudget zu sein [9]. Die geplanten Initiativen von CVP («Kostenbremse im Gesundheitswesen») und SP («Belastung durch die Krankenkassenprämien begrenzen») laufen klar auf dieses Ziel hinaus [21, 22]. Durch Anbindung völlig sachfremder Parameter (z.B. Einkommensanstieg oder prozentuale Fixwerte) werden die wesentlichen Gründe für den Kostenanstieg ausser Acht gelassen. Auch der Punkt «Massnahmen der Tarifpartner zur Steuerung der Kosten" aus dem geplanten 1. Massnahmenpaket zur Entlastung der OKP steuert in diese Richtung [23].

In der Schweiz beträgt der Bevölkerungsanstieg durchschnittlich 1,2\% pro Jahr, d.h., die Population hat in den letzten 10 Jahren um etwa $12 \%$ zugenommen. In Ländern wie Deutschland, Frankreich und den Niederlanden liegen diese Zahlen deutlich darunter (etwa 0\%, 0,4\% resp. 0,3\% jährlich) [24]. Zusätzlich ist zu erwarten, dass bis ins Jahr 2045 der Anteil der >65-Jährigen an der Bevölkerung, welche nachweislich die höchsten Gesundheitskosten verursachen, um über $84 \%$ zugenommen haben wird [25-27]. Hinzu kommen medizinische Innovationen, welche die Lebenserwartung weiter steigen lassen. Zu ergänzen ist auch, dass die Morbidität gewisser Erkrankungen (beispielsweise Melanom [28]) in den letzten Jahren deutlich zugenommen hat, was sich ebenfalls in höheren Kosten äussert.

All diese Faktoren werden auch in Zukunft zu weiter steigenden Gesundheitskosten führen, so dass sich Globalbudgets nur durch Rationierungen realisieren liessen. Treffender als mit der im Informationsorgan der Ärztegesellschaft des Kantons Bern publizierten Aussage [29] kann man dieses Faktum nicht umschreiben: «Wirtschaftlich wird am Ende nur noch derjenige arbeiten, der es schafft, sich von der Behandlung schwer kranker Menschen fernzuhalten.» 
$\mathrm{Zu}$ wenig Beachtung wird den auch qualitätsbezogenen Umfragen zum Gesundheitswesen geschenkt, beispielsweise dem Gesundheitsmonitor 2018 [30] oder dem CS-Sorgenbarometer 2017 [31], welche das gfs.bern jeweils im Auftrag erstellt. Diese zeigen: Die Schweizer Bevölkerung ist mit dem Gesundheitssystem hoch zufrieden, die Krankenkassenprämien sind breit akzeptiert und Sparmassnahmen sowie Experimente unerwünscht. Zudem zu erwähnen ist, dass die Ärzteschaft als Kostenverursacher an letzter Stelle genannt wird.

\section{Koordiniertes politisches Engagement der Ärzteschaft gefragt}

Symptomatisch für die aktuelle Debatte ist es, dass die aktiv berufstätige Ärzteschaft nicht in die Entscheidungsprozesse (z.B. «Expertengruppe») einbezogen wird. Auch konstruktive Vorschläge der FMH (Zulassungssteuerung [32], Kostendämpfungsmassnahmen [33]) werden übergangen. Denn in letzter Konsequenz haben wir Ärztinnen und Ärzte die qualitätsverschlechternden Massnahmen vor unseren Patientinnen und Patienten auch ethisch zu verantworten bzw. zu rechtfertigen.

Die Umstände und die Entwicklungen der letzten Jahre erfordern deswegen ein engeres und besser abgestimmtes Vorgehen, um ein politisch stärkeres Gewicht zu entwickeln. Herrscht Uneinigkeit, nutzt die Politik dies aus. Dafür muss sich die Solidaritätsund Kompromissbereitschaft unter den Ärztinnen und Ärzten sowie den einzelnen Fachgesellschaften verbessern.

Basierend auf Fakten statt auf Annahmen müssen durch Information der Patientinnen und Patienten sowie der politischen Entscheidungsträger die korrekten Weichenstellungen im Gesundheitswesen sichergestellt werden. Sie dürfen nicht zu Qualitätsabbau, Rationierungen und Zweiklassenmedizin führen.

\section{«If you focus on quality, you will reduce costs ..."}

Korrespondenz:

Dr. med. Daniel Mahler Dermapraxis Luzern

Alpenstrasse 9

6004 Luzern

Tel. 0414108383

dermapraxis-luzern[at]

hin.ch

Abschliessend sei die Aussage von Professor Jörg F. Debatin, dem ehemaligen Direktor des Universitätsklinikums Hamburg-Eppendorf, erwähnt [34]: «If you focus on quality, you will reduce costs, if you focus on costs, you will reduce quality.»
Literatur

1 Gesundheitskosten steigen auf über 80 Milliarden Franken. Medinside. 19.4.2018.

2 Neuberechnung der Gesamtgesundheitsausgaben 2015 Medienmitteilung BAG Nr. 2017-0082-D.

3 Health at a Glance 2017. OECD Indicators. http://www.oecd-ilibrary.org/social-issues-migration-health/health-at-aglance-2017_health_glance-2017-en.

4 Healthcare Access and Quality Index based on mortality from causes amenable to personal health care in 195 countries and territories, 1990-2015: a novel analysis from the Global Burden of Disease Study 2015. Lancet. 2017;390:231-66.

5 https://de.wikipedia.org/wiki/Liste_der_durchschnittlichen Lebenserwartung in den Staaten der Erde.

6 Es gibt zwar immer mehr Ärzte in der Schweiz - aber das hat einen Haken. Aargauer Zeitung. 28.3.2018.

7 http://www.oecdbetterlifeindex.org/de/topics/life-satisfactionde

8 https://www.aerzteblatt.de/nachrichten/34458/Gesundheit-hatentscheidenden-Einfluss-auf-Lebenszufriedenheit.

9 Kostendämpfungsmassnahmen-Bericht der Expertengruppe vom 24.8.2017.

10 Helsana-Report - Ausgabenentwicklungen in der Gesundheitsversorgung 12/16.

11 Hartwig J, et al. Baumolsche Kostenkrankheit im schweizerischen Gesundheitswesen. SÄZ. 2018;99:874-7.

12 Haushaltsbudgeterhebung 2008-2015. Bundesamt für Statistik.

$13 \mathrm{https} / / /$ taxfix.de/steuertipps/krankenversicherung-von-dersteuer-absetzen.

$14 \mathrm{http}: / /$ www.santesuisse.ch/de/details/content/standpunkt von_heinz_brand_im_tages_anzeiger_vom_30_september_ 2017_1308.

15 http://www.smartermedicine.ch.

16 https://www.fmh.ch/services/qualitaet/guidelines.html.

17 https://www.interpharma.ch/fakten-statistiken/1870-praemiensteigen-staerker-als-die-gesundheitskosten.

18 Vernehmlassung über einheitliche Finanzierung von ambulanten und stationären Behandlungen eröffnet. Medienmitteilung Parlament 05/18.

19 Arztpraxis: 70 Rappen pro Franken Ertrag sind Kosten. Medinside. 10.4.2018.

20 Oeschger C. TARCO ist bereit - Verhandlungen mit Tarifpartnern laufen weiter. SÄZ. 2018:99:536-53.

21 https://www.cvp.ch/de/initiative-fuer-tiefere-praemien-kostenbremse-im-gesundheitswesen.

22 https://www.sp-ps.ch/de/publikationen/espress/belastungdurch-die-krankenkassenpraemien-begrenzen.

23 Kostendämpfungsmassnahmen zur Entlastung der obligatorischen Krankenpflegeversicherung (OKP): 1. Massnahmenpaket für die Vernehmlassung vom 14.9.2018.

24 http://countrymeters.info.

25 Gesundheitswesen Schweiz 2013. Credit Suisse Global Research 2013.

26 Gesundheitswesen: Wachstumsmarkt unter Kostendruck Credit Suisse; Swiss issues Konjunktur; März 2017.

2710 Millionen Einwohner im Jahr 2045. NZZ. 22.6.2015

28 SGDV - Nationale Hautkrebskampagne 2018, Presseunterlagen.

29 "Wirtschaftlich ist, wer sich von kranken Menschen fernhält». doc.be 06/17.

30 Gesundheitsmonitor 2018: Experimente unerwünscht, aber wachsende Ansprüche an die Versorgung. Interpharma.
31 CS-Sorgenbarometer 2017: Altersvorsorge wird zur Topsorge. Credit Suisse.

32 Die FMH will über nationale Qualitätskriterien steuern. Medienmitteilung 9.5.2018.

33 Wille N, et al. Die FMH zur Dämpfung des Kostenanstiegs im Gesundheitswesen. SÄZ. 2018;99:224-6.

34 https://www.vzk.ch/gb2017/zwischenruf. 